\title{
Breakdown of Stabilization of Atoms Interacting with Intense, High-Frequency Laser Pulses
}

\author{
N. J. Kylstra, ${ }^{1}$ R. A. Worthington, ${ }^{1}$ A. Patel, ${ }^{2}$ P. L. Knight, ${ }^{2}$ J. R. Vázquez de Aldana, ${ }^{3}$ and L. Roso ${ }^{3}$ \\ ${ }^{1}$ Department of Physics, Science Laboratories, University of Durham, Durham DH1 3LE, United Kingdom \\ ${ }^{2}$ Optics Section, Blackett Laboratory, Imperial College, London SW7 2BZ, United Kingdom \\ ${ }^{3}$ Departamento de Física Aplicada, Universidad de Salamanca, E-37008 Salamanca, Spain
}

(Received 13 April 2000)

\begin{abstract}
An analysis of the influence of the magnetic field of an intense, high-frequency laser pulse on the stabilization of an atomic system is presented. We demonstrate that at relatively modest intensities the magnetic field can significantly alter the dynamics of the system. In particular, a breakdown of stabilization occurs, thereby restricting the intensity regime in which the atom is relatively stable against ionization. Counterpropagating pulses do not negate the detrimental effects of the magnetic field. We compare our quantum mechanical results with classical Monte Carlo simulations.
\end{abstract}

PACS numbers: $32.80 . \mathrm{Fb}, 32.80 . \mathrm{Rm}, 42.50 . \mathrm{Hz}$

Theoretical studies of atoms interacting with highfrequency intense laser pulses have predicted a significant decrease in the ionization probability with increasing laser intensity. This phenomenon is referred to as atomic stabilization, and has been extensively studied over the past decade [1]. Many aspects of this phenomenon can be understood by performing a Kramers-Henneberger $(\mathrm{KH})$ transformation to the rest frame of a classical electron in the laser field. In particular, by developing a highfrequency Floquet theory in the KH frame [2], stabilization can be seen to have its origin in the rapid quiver motion of the atomic electron in the laser field. This allows the electron dynamics to be described by an effective potential that, on average, localizes the electron away from the vicinity of the nucleus. Subsequent $a b$ initio Floquet calculations confirmed that ionization rates decrease with increasing intensity in a high-frequency field [3]. By directly integrating the time-dependent Schrödinger equation numerically, simulations in one [4] and three dimensions [5] demonstrated reductions in the ionization probability with increasing laser intensity when an atom interacts with realistic laser pulses having a finite duration. Further work has been carried out in order to elucidate the effects of the pulse shape and duration $[6,7]$. We also note that evidence of atomic stabilization of Rydberg states has been observed experimentally [8].

In the above-mentioned theoretical studies, the magnetic component of the laser pulse was neglected. However, as the laser intensity increases, relativistic effects that alter the stabilization dynamics become important. Classical Monte Carlo simulations have indicated that the magnetic field pushes the electron in the laser pulse propagation direction, reducing the degree of stabilization [9]. Relativistic wave equations have also been considered within the context of reduced dimensional models $[10,11]$. However, it is also of interest to study the effects that are neglected in the dipole approximation by using the fully space- and time-dependent vector potential in the nonrelativistic Schrödinger equation [12]. For atomic hydrogen, this results in the cylindrical symmetry of the system being broken, thereby requiring a fully three-dimensional calculation to be carried out for extremely high laser intensities. This is a computationally demanding task. However, a two-dimensional model atom contains the essential physics [11], allowing studies of laser-atom interactions beyond the dipole approximation to be readily carried out [13]. In this Letter we investigate the laser-atom dynamics in the stabilization regime for a two-dimensional model atom without employing the dipole approximation. We demonstrate that stabilization breaks down at relatively modest laser intensities, i.e., at intensities where the laser-atom interaction can be described by the nonrelativistic Schrödinger equation. In particular, we show that the dipole approximation leads to survival probabilities which are too large because the magnetic field, neglected in the dipole approximation, strongly influences the stabilization dynamics. By comparing our results with those obtained from classical Monte Carlo simulations, we confirm the validity of classical approaches in describing atoms interacting with short, highintensity, and high-frequency laser pulses.

Our results have been obtained using a two-dimensional model atom which is described by the time-dependent Schrödinger equation

$$
i \frac{\partial}{\partial t} \Psi(\mathbf{r}, t)=\left[\frac{1}{2}\left(\mathbf{p}+\frac{1}{c} \mathbf{A}(\eta)\right)^{2}+V(r)\right] \Psi(\mathbf{r}, t),
$$

with $\mathbf{r}=(x, z)$. The spin of the electron is neglected and we use atomic units (a.u.) throughout. The laser field is described classically by the fully spatially dependent vector potential

$$
\mathbf{A}(\eta)=\frac{E_{0} c}{\omega} \hat{\mathbf{x}} f(\eta) \sin \eta .
$$

The function $f(\eta)$ is the laser pulse envelope, which is taken to be trapezoidal, and $\eta=\omega(t-z / c)$ so that the laser pulse travels in the positive $z$ direction and $c$ is the speed of light. We use a smoothed two-dimensional 
Coulomb potential

$$
V(r)=-\frac{1}{\sqrt{r^{2}+a^{2}}} .
$$

The smoothing parameter $a$ is used to avoid problems in two dimensions associated with the singularity at $r=0$. We have set $a=0.80$ so that the ground state has the same binding energy as hydrogen, i.e., -0.50 . The potential supports an infinite number of Rydberg series of bound states. Equation (1) is numerically integrated on a uniform grid using either a split operator or a Crank-Nicholson method. Both widely used approaches are easily modified to account for the spatially dependent vector potential.

The probability that the atom does not ionize during the laser pulse is determined by projecting the wave function at the end of the laser pulse onto the bound field-free states of the atom,

$$
P_{\text {survival }}=\sum_{n, m}\left|\left\langle\psi_{n m} \mid \Psi(\tau)\right\rangle\right|^{2},
$$

where $\tau$ is the pulse duration. The field-free states $\left|\psi_{n m}\right\rangle$ are labeled by their principal quantum number $n$ and azimuthal quantum number $m$. In cylindrical coordinates, $\psi_{n m}(\mathbf{r})=(2 \pi)^{1 / 2} R_{n m}(r) \exp (-i m \phi)$ so that the radial functions are solutions of the differential equation

$$
\left[-\frac{1}{2 r} \frac{\partial}{\partial r}\left(r \frac{\partial}{\partial r}\right)+\frac{m^{2}}{2 r^{2}}+V(r)-E_{n, m}\right] R_{n m}(r)=0 .
$$

To understand the influence of the magnetic field, we also performed calculations in the dipole approximation by setting $\eta=\omega t$ in Eq. (2) for the vector potential.

In the upper plots of Fig. 1, the ionization probability is shown as a function of the peak electric field strength of the laser, $E_{0}$, for angular frequencies of $\omega=1$ (left) and $\omega=2$ (right). As indicated in the plots, the dashed lines are nondipole (NDP) results and solid lines are the results of the dipole (DP) approximation. A trapezoidal pulse with three-cycle turn-on (turn-off) and six-cycle constant intensity was used. From the results, it is evident that the dipole approximation breaks down when the electric field $E_{0} \gtrsim 10$ for $\omega=1$. However, when $\omega=2$ it remains a good approximation up to $E_{0}=20$, the maximum field strength considered here.

In the lower plots of Fig. 1, the populations in the ground state and in the even parity bound states are shown. For a twelve-cycle pulse and $\omega=1$, nearly all of the surviving population returns to the ground state for $E_{0} \lesssim 5$. This is an indication of the so-called "adiabatic stabilization" of the atom. When $E_{0} \gtrsim 10$, the evolution of the system is highly nonadiabatic, and in the dipole approximation there is significant population transfer ("shake up") to the excited states. Note that, in this "dynamical stabilization" regime, only even parity states are populated, i.e., the evolution of the system can be described to a good approximation by an effective time-averaged potential in

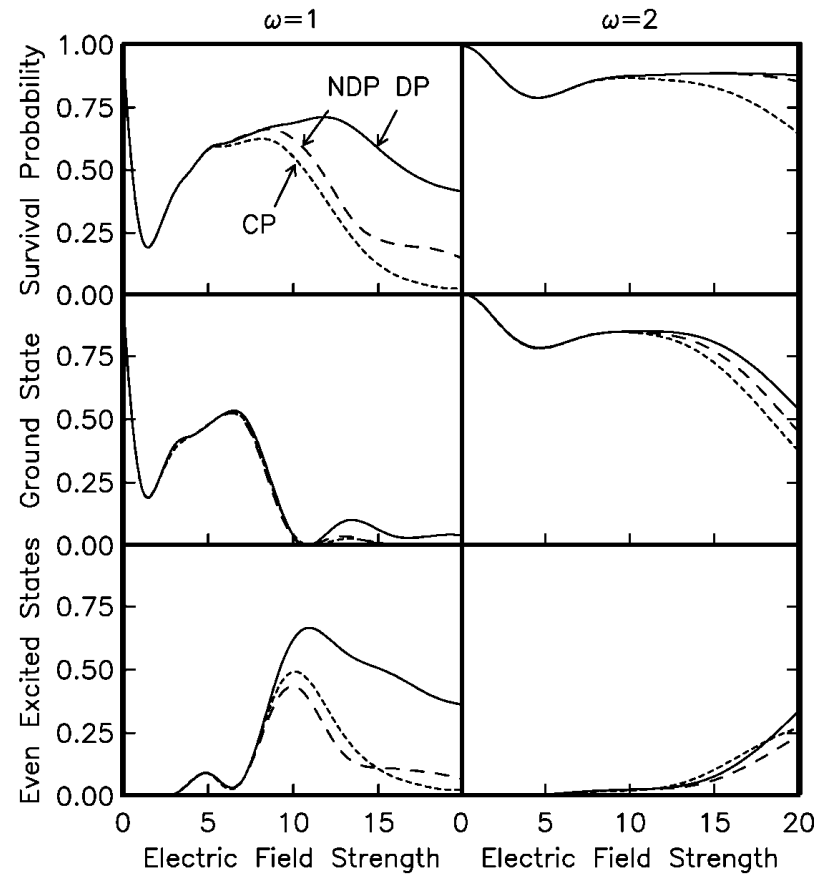

FIG. 1. The upper plot shows the survival probability as a function of the maximum electric field strength, $E_{0}$, of the laser. On the left, results are shown for an angular frequency of $\omega=1$ while, on the right, $\omega=2$. A trapezoidal pulse with a three-cycle turn-on (turn-off) and six-cycle constant intensity was used. In the lower two plots, the populations in the ground state and in the even parity excited states are shown. The dipole (DP) results are given by the solid curves, nondipole (NDP) results are given by the dashed curves while the dotted curves correspond to results for two counterpropagating $(\mathrm{CP})$ pulses.

the $\mathrm{KH}$ frame [7]. For the nondipole case, the surviving population moves progressively to the odd parity states as the dipole approximation breaks down. When $\omega=2$, adiabaticity is maintained up to $E_{0} \simeq 10$. We have performed additional calculations for a range of pulse durations. These results, which will be presented elsewhere, show that the degree of adiabaticity of the laser-atom interaction does not modify significantly the breakdown of the dipole approximation.

The fact that the dipole approximation breaks down at relatively moderate intensities, and that the dipole approximation improves as the frequency increases, can be understood by using the Lorentz force equations. To lowest order in $1 / c$ for a free electron initially at rest, the force exerted on the electron and the velocity of the electron in the laser pulse are

$$
\begin{gathered}
v_{x}(t)=\left.\frac{A_{x}(\eta)}{c}\right|_{z=0}, \\
F_{z}(t)=-\left.\frac{v_{x}(t)}{c} \frac{\partial A_{x}(\eta)}{\partial z}\right|_{z=0}=\left.\frac{1}{2 c^{3}} \frac{\partial A_{x}^{2}(\eta)}{\partial t}\right|_{z=0}, \\
v_{z}(t)=\frac{1}{2 c^{3}} A_{x}^{2}(\omega t) .
\end{gathered}
$$


Note that the magnetic field induces a velocity in the propagation direction which is never negative. This velocity is composed of a drift term and an oscillating term. In addition, the displacement of the electron in the propagation direction increases as the pulse duration increases. By integrating Eq. (8), this simple model implies that the dipole approximation is expected to fail when the the displacement of the electron arising from its "figure eight" motion due to the magnetic field becomes comparable to the size of the initial wave packet, i.e., $E_{0}^{2} / c \omega^{3} \sim 1$. This is confirmed by our calculations. When this occurs, the total displacement of a classical electron in the laser field is proportional to $\tau E_{0}^{2} / c \omega^{2}$, where $\tau$ is taken to be approximately the duration of the flat part of the pulse. We emphasize that for $E_{0}$ fixed, decreasing $\omega$ will result in an eventual breakdown of the dipole approximation in this high-intensity regime.

One might imagine that the detrimental effect of the magnetic field can be eliminated by having the atom interact with two counterpropagating laser pulses. Taking a vector potential of the form

$$
\mathbf{A}\left(\eta, \eta^{\prime}\right)=\frac{c E_{0}}{2 \omega} \hat{\mathbf{x}}\left(\sin \eta^{\prime}+\sin \eta\right)
$$

with $\eta^{\prime}=\omega(t+z / c)$, will result in fixed points in space where the magnetic field vanishes, while the electric field does not. In particular, at $z=0$ the electric field oscillates between $-E_{0}$ and $E_{0}$, but the magnetic field vanishes for all times. It therefore seems reasonable that for a standing wave the effect of the magnetic field would be minimized and stabilization maintained. Near $z=0$ the magnetic field, of course, does not vanish.

We have calculated the time evolution of the initial ground state of our two-dimensional model atom interacting with two linearly polarized counterpropagating laser pulses described by the vector potential

$$
\mathbf{A}\left(\eta, \eta^{\prime}\right)=\frac{c E_{0}}{2 \omega} \hat{\mathbf{x}}\left[f\left(\eta^{\prime}\right) \sin \eta^{\prime}+f(\eta) \sin \eta\right] .
$$

In Fig. 2, plots of the wave function probability density are shown after 3, 6, and 9 cycles of the counterpropagating pulses (right). Results for the dipole approximation are also shown (left). Here $E_{0}=15, \omega=1$ and again $f(\eta)$ describes a pulse with a three-cycle linear turn-on (turnoff) and six cycles of constant intensity. Clearly magnetic field effects strongly influence the stabilization dynamics even for counterpropagating pulses. From Fig. 1, it is seen that in fact the counterpropagating pulses result in survival probabilities that are smaller than when a single pulse is used. During the laser turn-on, the magnetic fields do not exactly cancel to lowest order in $1 / c$. This leads to a drift velocity that is negative for $z$ negative and positive for $z$ positive. In addition, after the turn-on and to lowest order in $z / c$, the force along the $z$ direction on the electron
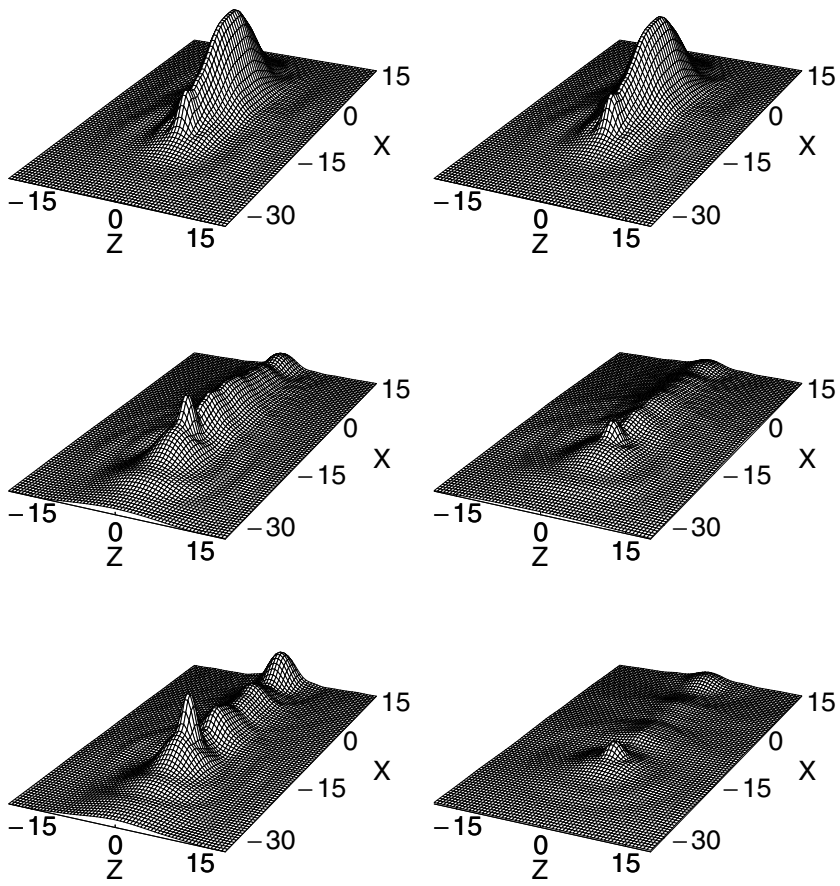

FIG. 2. Plots of the probability density of the wave function for linearly polarized (along the $x$ axis), counterpropagating pulses (right). Dipole approximation results are also shown (left). These snapshots are taken after three (top), six (middle) and nine (bottom) cycles. The pulse shapes are the same as in Fig. 1, the maximum electric field strength of the pulses is $E_{0}=15$ and the angular frequency is $\omega=1$.

in the laser pulse (and in the absence of the nucleus) is given by

$$
\begin{gathered}
F_{z}(t, z)=\frac{v_{x}(t)}{c^{3}} z\left[\frac{\partial^{2} A_{x}\left(\eta, \eta^{\prime}\right)}{\partial t^{2}}\right]_{z=0}, \\
v_{x}=\left.\frac{A_{x}\left(\eta, \eta^{\prime}\right)}{c}\right|_{z=0} .
\end{gathered}
$$

From the above equations, we can see that the force will always act such that it drives the electron away from the origin. This is also detrimental to stabilization. Note that, while the force $F_{z}(t, z)$ is a factor $\sim z \omega / c$ smaller than $F_{z}(t)$ defined in Eq. (7), it is nevertheless comparable to the force of the time-averaged potential in the KH frame that binds the electron (in the dipole approximation). By considering different laser pulse turn-ons, we have verified that the force given by Eq. (11) is large enough to destabilize the atom.

Finally, we compare our results with classical Monte Carlo simulations. Previous classical simulations have indicated a breakdown of stabilization due to the magnetic field component [9]. However, the applicability of approaches based on classical mechanics has remained an open question. Our results are shown in Fig. 3. The ensemble of initial conditions was obtained in a manner analogous to the microcanonical distribution used for the Kepler problem [9]. Typically, 500 trajectories were calculated for each value of $E_{0}$. It is seen that the results of the 


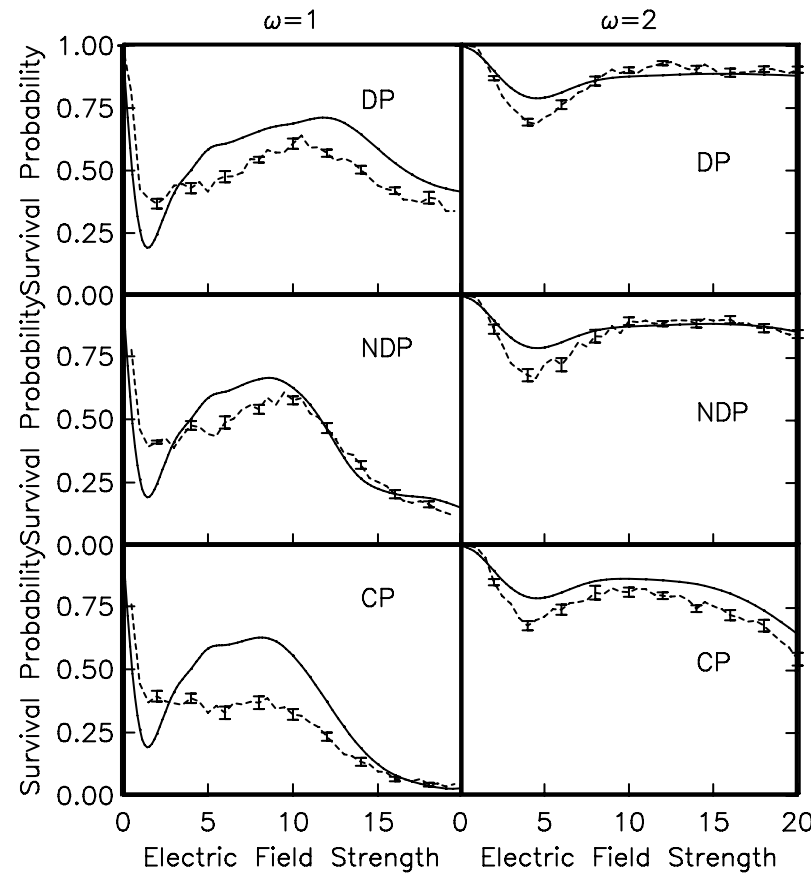

FIG. 3. Quantum (solid curves) and classical Monte Carlo (dotted curves) survival probabilities as a function of the maximum electric field strength, $E_{0}$, of the laser. On the left, results are shown for an angular frequency of $\omega=1$ while, on the right, $\omega=2$. The remaining pulse parameters are the same as in Fig. 1. Dipole (DP), nondipole (NDP), and counterpropagating pulses (CP) results are shown. Error estimates are given for the Monte Carlo results.

quantum and classical calculations demonstrate a remarkable agreement for each of the cases considered. Evidently, aspects of the wave packet dynamics of the system in the high-frequency, high-intensity regime can be described to a good approximation by using classical mechanics.

We have presented the results of numerical simulations involving a two-dimensional model atom interacting with an intense laser pulse that includes the magnetic field of the laser pulse. We have clearly shown that the magnetic field induces a drift along the propagation direction that is sufficient, even for moderate intensities, to disrupt the electron dynamics that are required for atomic stabilization. By considering the Lorentz force on the electron in the propagation direction of the laser pulse, it can be seen that even a small drift velocity, integrated over the duration of the laser pulse, is sufficient to modify the simple oscillating motion along the polarization direction, thereby leading to a breakdown of stabilization. It should be stressed that, for the laser frequencies and intensities considered here, the dynamics of the electron in the laser field is essentially nonrelativistic. Indeed, for the highest field strength considered, $E_{0}=20$, the ratio of the ponderomotive energy of the electron in the laser field to its rest mass energy is small: $E_{0}^{2} /\left(4 \omega^{2} c^{2}\right)=5 \times 10^{-3}$. In addition, for the laser field parameters considered, the laser coupling of the electron spin degrees of freedom is expected to have a negligible influence on the stabilization dynamics.

In conclusion, our results indicate that important revisions are needed of existing stabilization models so that the effect of the magnetic field is properly accounted for.

This work has been supported by the UK Engineering and Physical Sciences Research Council (EPSRC) and the Spanish Dirección General de Enseñanza Superior e Investigación Científica (Grant No. PB98-0268).

[1] For reviews, see C. J. Joachain, M. Dörr, and N. J. Kylstra, Adv. At. Mol. Opt. Phys. 42, 225 (1999); M. Protopapas, C. H. Keitel, and P.L. Knight, Rep. Prog. Phys. 60, 389 (1997).

[2] M. Pont, N. R. Walet, M. Gavrila, and C. W. McCurdy, Phys. Rev. Lett. 61, 939 (1988); M. Pont, N. R. Walet, and M. Gavrila, Phys. Rev. A 41, 477 (1990); M. Pont and M. Gavrila, Phys. Rev. Lett. 65, 2362 (1990).

[3] M. Dörr, R. M. Potvliege, D. Proulx, and R. Shakeshaft, Phys. Rev. A 43, 3729 (1991).

[4] Q. Su, J.H. Eberly, and J. Javanainen, Phys. Rev. Lett. 64, 862 (1990); C. K. Law, Q. Su, and J. H. Eberly, Phys. Rev. A 44, 7844 (1991).

[5] K. C. Kulander, K. J. Schafer, and J. L. Krause, Phys. Rev. Lett. 66, 2601 (1991).

[6] V.C. Reed, P. L. Knight, and K. Burnett, Phys. Rev. Lett. 67, 1415 (1991); R. M. A. Vivirito and P. L. Knight, J. Phys. B 28, 4357 (1995).

[7] A. S. Patel, N. J. Kylstra, and P. L. Knight, J. Phys. B 32, 5759 (1999).

[8] M. P. de Boer, J. H. Hoogenraad, R. B. Vrijen, L. D. Noordam, and H. G. Muller, Phys. Rev. Lett. 71, 3263 (1993); see also B. Piraux and R. M. Potvliege, Phys. Rev. A 57, 5009 (1998).

[9] J. Grochmalicki, M. Lewenstein, and K. Rza̧żewski, Phys. Rev. Lett. 66, 1038 (1991); C. H. Keitel and P. L. Knight, Phys. Rev. A 51, 1420 (1995); see also T. Katsouleas and W. B. Mori, Phys. Rev. Lett. 70, 1561 (1993).

[10] M. Protopapas, C. H. Keitel, and P. L. Knight, J. Phys. B 29, L591 (1996); N. J. Kylstra, A. M. Ermolaev, and C. J. Joachain, J. Phys. B 30, L449 (1997).

[11] U. W. Rathe, C. H. Keitel, M. Protopapas, and P. L. Knight, J. Phys. B 30, L531 (1997).

[12] A. Bugacov, M. Pont, and R. Shakeshaft, Phys. Rev. A 48, R4027 (1993); O. Latinne, C. J. Joachain, and M. Dörr, Europhys. Lett. 26, 333 (1994); R. M. Potvliege, Laser Phys. 10, 143 (2000).

[13] J. R. Vázquez de Aldana and L. Roso, Opt. Express 5, 144 (1999); Phys. Rev. A 61, 043403 (2000). 